\title{
Produção de mudas de Leucaena leucocephala com uso de fertilizante mineral e
}

\section{organomineral}

\author{
Production of Leucaena leucocephala seedlings using mineral and organic mineral fertilizer \\ Producción de plántulas de Leucaena leucocephala empleando fertilizantes mineral y
}

organomineral

Recebido: 18/01/2021 | Revisado: 24/02/2021 | Aceito: 09/02/2021 | Publicado: 15/02/2021

\author{
Alan de Sousa \\ ORCID: https://orcid.org/0000-0002-5720-9521 \\ Universidade Federal do Piauí, Brasil \\ E-mail: alandesousa161@gmail.com \\ Rafael Felippe Ratke \\ ORCID: https://orcid.org/0000-0001-6930-3913 \\ Universidade Federal de Mato Grosso do Sul, Brasil \\ E-mail: rafael.ratke@ufms.br \\ Jonas Wilson Parente Vieira \\ ORCID: https://orcid.org/0000-0002-2839-4975 \\ Universidade Federal do Piauí, Brasil \\ E-mail: jonas1k10@hotmail.com \\ Fabiana Vieira de Souza \\ ORCID: https://orcid.org/0000-0002-8815-2257 \\ Universidade Federal do Piauí, Brasil \\ E-mail: fabiana.souza.fs255@gmail.com \\ Alan Mario Zuffo \\ ORCID: https://orcid.org/0000-0001-9704-5325 \\ Universidade Federal de Mato Grosso do Sul, Brasil \\ E-mail: alan_zuffo@hotmail.com \\ Jorge González Aguilera \\ ORCID: https://orcid.org/0000-0002-7308-0967 \\ Universidade Federal de Mato Grosso do Sul, Brasil \\ E-mail: j51173@yahoo.com
}

\begin{abstract}
Resumo
A adubação fosfatada promove o desenvolvimento de mudas florestais. Dessa forma, os estudos de meios de adição de fósforo (P) orgânico e inorgânico são necessários para promover a qualidade de mudas de leucena (Leucaena leucocephala). Diante disso, objetivou-se avaliar o crescimento inicial e qualidade das mudas de leucena submetidas a doses de fertilizantes mineral e organomineral. As plantas de leucena foram adubadas com dois fertilizantes, sendo: mono-amônio-fosfato (MAP) (9\% de $\mathrm{N}$ e $48 \%$ de $\mathrm{P}_{2} \mathrm{O}_{5}$ ), e um organomineral (OM) (11\% de $\mathrm{N}$ e $26.6 \% \mathrm{P}_{2} \mathrm{O}_{5}, 18,2 \%$ de Carbono Orgânico) e quatro doses de $\mathrm{P}_{2} \mathrm{O}_{5}\left(0 ; 10 ; 20\right.$ e $\left.30 \mathrm{mg} \mathrm{kg}^{-1}\right)$, com três repetições. Aos 60 dias após o plantio avaliou-se a altura de plantas (AP), diâmetro do caule (DC), biomassa da parte áerea (BMPA), biomassa da raiz (BMR), massa seca da parte áerea (MSPA), massa seca da raiz (MSR), quociente de robustez (ROB) e Índice de Qualidade de Dickson (IQD). As médias de AP, DC e IQD não foram influenciadas pelas fontes e doses de P, porém as médias BMPA, BMR, MSPA, MSR e ROB apresentaram influência significativa para fontes e doses de P. O fertilizante mineral promove melhor efeito nas variaveis da biomassa da parte aerea e o organomineral favorece a biomasssa da raiz. Houve correções positivas entre os parametros avaliado de muda leucena: BMPA, BMR, MSPA, MSR e IQD, e a ROB apresentou correlação negativa com DC.
\end{abstract}

Palavras-chave: Leucena; Índice de Qualidade de Dickson; Rede de correlações de Pearson.

\begin{abstract}
Phosphate fertilization promotes the development of forest seedlings. Thus, studies on means of adding organic and inorganic phosphorus $(\mathrm{P})$ are necessary to promote the quality of Leucena (Leucaena leucocephala) seedlings. Therefore, the objective was to evaluate the initial growth and quality of Leucena seedlings submitted to doses of mineral and organic mineral fertilizers. The Leucena plants were fertilized with two fertilizers being: monoammonium-phosphate (MAP) $\left(9 \% \mathrm{~N}\right.$ and $\left.48 \% \mathrm{P}_{2} \mathrm{O}_{5}\right)$ and an organic mineral $(\mathrm{OM})(11 \% \mathrm{~N}$ and $26.6 \% \mathrm{P} 2 \mathrm{O} 5,18.2 \%$ Organic Carbon) and four doses of $\mathrm{P}_{2} \mathrm{O}_{5}\left(0 ; 10 ; 20\right.$ and $\left.30 \mathrm{mg} \mathrm{kg}^{-1}\right)$, with three repetitions. At 60 days after planting were evaluated: plant height (AP), stem diameter (DC), aerial biomass (BMPA), root biomass (BMR), aerial dry mass (MSPA), dry mass of the root (MSR), robustness quotient (ROB) and Dickson's Quality Index (IQD). The means of $\mathrm{AP}, \mathrm{DC}$ and IQD were not influenced by the sources and doses of P, but the means BMPA, BMR, MSPA, MSR and
\end{abstract}


ROB showed significant influence for sources and doses of $\mathrm{P}$. The mineral fertilizer promotes better effect in the variables of the biomass of the aerial part and the organic mineral favors the biomass of the root. There were positive corrections between the parameters evaluated for Leucena seedling: BMPA, BMR, MSPA, MSR and IQD, and ROB showed a negative correlation with DC.

Keywords: Leucena; Dickson's Quality Score; Pearson's correlation network.

\section{Resumen}

La fertilización con fosfato promueve el desarrollo de plántulas forestales. Por lo tanto, son necesarios estudios sobre la forma de agregar fósforo $(\mathrm{P})$ orgánico e inorgánico para promover la calidad de las plántulas de leucena (Leucaena leucocephala). Por tanto, el objetivo fue evaluar el crecimiento inicial y la calidad de plántulas de leucena sometidas a dosis de fertilizantes minerales y organominerales. Las plantas de leucena fueron fertilizadas con dos fertilizantes, siendo: mono-fosfato amónico (MAP) $\left(9 \% \mathrm{~N}\right.$ y $\left.48 \% \mathrm{P}_{2} \mathrm{O}_{5}\right)$ y un organomineral, $(\mathrm{OM})\left(11 \% \mathrm{~N}\right.$ y $26.6 \% \mathrm{P}_{2} \mathrm{O}_{5}, 18.2 \%$ Carbono orgánico) y cuatro dosis de $\mathrm{P}_{2} \mathrm{O}_{5}\left(0 ; 10 ; 20\right.$ y $\left.30 \mathrm{mg} \mathrm{kg}^{-1}\right)$, con tres repeticiones. A los 60 días después de la siembra, se evaluó la altura de la planta (AP), el diámetro del tallo (DC), la biomasa aérea (BMPA), la biomasa radicular (BMR), la masa seca aérea (MSPA), la masa seca de la raíz (MSR), el cociente de robustez (ROB) y el índice de calidad de Dickson (IQD). Las medias de la altura de la planta, el diámetro del tallo y el IQD no fueron influenciadas por las fuentes y dosis de P, pero las medias BMPA, BMR, MSPA, MSR y ROB mostraron una influencia significativa para las fuentes y dosis de P. El fertilizante mineral promueve un mejor efecto en las variables de la biomasa de la parte aérea y la organomineral favorecen la biomasa de la raíz. Hubo correcciones positivas entre los parámetros evaluados para plántula de leuceno: BMPA, BMR, MSPA, MSR e IQD, y ROB mostró una correlación negativa con DC.

Palabras clave: Leucena; Índice de Cualidad de Dickson; Red de correlación de Pearson.

\section{Introdução}

A leucena é uma espécie originária da América Central, e muito utilizada na região semiárido do Brasil (Oliveira et al., 2016). Essa planta é uma leguminosa com grande diversidade de usos e por isso vem sendo bastante utilizada em nosso país, principalmente na região Nordeste por apresentar um crescimento rápido, tolerância à seca, e possuir alta capacidade de rebrota, sendo bastante empregada como adubação verde (Silva et al., 2015). Outra característica marcante desta espécie é seu uso na pecuária como banco de proteína, podendo ser utilizada como pastejo direto, fornecimento de alimento verde no cocho, silagem ou também o enriquecimento de silagem em gramíneas como também na produção de feno na região do semiárido brasileira (Sousa, 2005)

Entretanto, para que ocorra produção de mudas de qualidades, existe diversos fatores limitantes, entres elas, a adubação (Oliveira et al., 2016). Nas últimas décadas o fósforo tem sido o elemento mais crítico nas adubações, em razão do alto grau de interação com o substrato (Raij, 2011). O aumento da disponibilidade de fósforo para as plantas pode ser obtido mediante o manejo correto da adubação fosfatada, com ênfase na fonte utilizada e no modo de aplicação mais adequado para substrato com diferentes capacidades de adsorção do elemento (Corrêa et al., 2005). O fósforo participa diretamente nos açúcares-fosfato, ácidos nucléicos, nucleotídeos, coenzimas, fosfolipídeos, ácido fítico e manifesta papel principal nas reações de produção de ATP para as plantas (Taiz et al., 2017).

Os fertilizantes organominerais $(\mathrm{OM})$ vem se destacando na adição de matéria orgânica ao solo e na fertilização das culturas, contribuindo para o aumento da disponibilidade de fósforo no substrato, a partir do fornecimento de fenóis, advindos da liberação residual de ácidos húmicos (Almeida Júnior et al., 2011). Segundo Tejada, Benitez, e Gonzalez, (2002), o uso do fertilizante organomineral reduz os custos com adubação e permite o suprimento de nutrientes minerais e matéria orgânica ao solo.

Para atender a demanda nutricional nas fases iniciais das plantas, e reduzir as perdas de nutriente, garantindo um bom efeito residual do fertilizante, sugerem-se o uso do fertilizante organomineral (Baron, Benitez e Gonzalez, 1995). Segundo Medeiros (1992), afirma que uma das fases mais críticas para o estabelecimento das espécies florestais e frutíferas é a fase de produção de mudas, tanto em sua qualidade como em quantidade.

A fertilização dos substratos com adubos minerais e húmus são necessárias para melhorar o desenvolvimento de 
mudas de espécies florestais (Santos et al., 2019). O uso de fontes minerais e orgânicas para o fornecimento de nutrientes para as mudas são importantes para favorecer o desenvolvimento das mudas, e torná-las viáveis para sua implementação no campo. Diante disso, objetivou-se avaliar o crescimento inicial e qualidade das mudas de leucena submetidas a doses de fertilizantes mineral e organomineral.

\section{Metodologia}

O experimento foi realizado em casa de vegetação na Estação Experimental Agronômica da Universidade Federal do

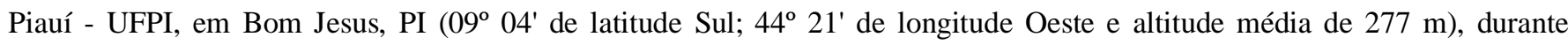
novembro de 2016 a fevereiro de 2017. O clima da região, segundo classificação de Köppen, é do tipo quente e semiúmido (Aw), com precipitação média de 900 a $1.200 \mathrm{~mm} /$ ano e duas estações bem definidas, a seca no período de maio a outubro e a chuvosa de novembro a abril, com temperatura média de 26,5年 (Alvares et al., 2014).

O solo utilizado no experimento foi um Latossolo Amarelo distrófico típico de textura franco-arenosa, coletado em uma área nativa de Caatinga na camada superficial de 0,20 - 0,40 m profundidade. Antes da instalação do experimento, o solo coletado foi homogeneizado manualmente com auxílio de uma enxada, posteriormente retirou-se uma amostra com cerca de $500 \mathrm{~g}$ para caracterização quanto aos atributos químicos e físicos seguindo a metodologia de Silva (2011). Os valores encontrados foram: $\mathrm{pH}$ em $\mathrm{H}_{2} \mathrm{O}=5,0 ; \mathrm{P}=4,08 \mathrm{mg} \mathrm{dm}{ }^{-3} ; \mathrm{K}=23,40 \mathrm{mg} \mathrm{dm}-3 ; \mathrm{Ca}^{2+}=0,0 \mathrm{cmol}_{\mathrm{c}} \mathrm{dm}^{-3} ; \mathrm{Mg}^{2+}=0,0 \mathrm{cmol}_{\mathrm{c}} \mathrm{dm}^{-3}$; $\mathrm{Al}^{3+}=0,30 \mathrm{cmol}_{\mathrm{c}} \mathrm{dm}-3 ; \mathrm{H}^{+} \mathrm{Al}=5,61 \mathrm{cmol}_{\mathrm{c}} \mathrm{dm}^{-3} ; \mathrm{V}=1,1 \% ; \mathrm{CTC}=3,75 \mathrm{cmol}_{\mathrm{c}} \mathrm{dm}^{-3} ;$ matéria orgânica $=13,2 \mathrm{~g} \mathrm{~kg}^{-1} ;$ areia: $673 \mathrm{~g} \mathrm{~kg}^{-1}$, silte: $52 \mathrm{~g} \mathrm{~kg}^{-1} \mathrm{e}$ argila: $275 \mathrm{~g} \mathrm{~kg}^{-1}$.

A necessidade de correção da acidez do solo foi calculada pelo método da elevação da saturação por base à $50 \%$ (Sousa e Lobato, 2004), o que correspondeu à dose de 4,1 $\mathrm{t} \mathrm{ha}^{-1}$ ou 2,05 $\mathrm{g} \mathrm{L}^{-1}$ de solo. Utilizou-se calcário agrícola com PRNT de $92 \%$ (35\% de $\mathrm{CaO}$ e $6 \%$ de $\mathrm{MgO}$ ). Após a calagem, o solo foi homogeneizado, irrigado e mantido incubado por 30 dias.

O delineamento experimental utilizado foi o inteiramente casualizado, disposto em esquema fatorial $2 \times 4$, sendo os fatores constituídos por dois 2 fertilizantes, sendo mono-amônio-fosfato ( $\mathrm{MAP}-9 \%$ de $\mathrm{N}$ e $48 \%$ de $\mathrm{P}_{2} \mathrm{O}_{5}$ ) e um organomineral (OM: esterco de aviário de frangos mais adição de MAP, concentração final OM: - 9\% de $\mathrm{N}$ e $26.6 \% \mathrm{P}_{2} \mathrm{O}_{5}, 18,2 \%$ de Carbono Orgânico) e quatro doses de $\mathrm{P}_{2} \mathrm{O}_{5}\left(0 ; 10 ; 20\right.$ e $\left.30 \mathrm{mg} \mathrm{kg}^{-1}\right)$, com três repetições

As sementes utilizadas no experimento foram coletadas de árvores matrizes nas proximidades do campus. Após a coleta, as sementes foram previamente selecionadas, levando-se em conta a tamanho e, então, submetidas ao tratamento prégerminativo para superação da dormência. As sementes foram imersas em água a $80^{\circ} \mathrm{C}$ por 5 min para a quebra de dormência (Decker et al., 2011). Em seguida, dez sementes foram semeadas $\pm 2 \mathrm{~cm}$ de profundidade por vaso e, após o estabelecimento, realizou-se o desbaste deixando três plantas por vaso. Cada unidade experimental foi composta por três mudas de leucena semeadas em vasos de polietileno de 3,8 litros. Após 7 dias da germinação da leucena, deixou-se apena uma planta por vaso.

O teor de água do substrato foi mantido próximo da capacidade de retenção de água com irrigações diárias mantendose o nível de $80 \%$ da capacidade de vaso, medindo-se o peso de vaso saturado e insaturado através de uma balança de precisão $(\mathrm{P}=0,001 \mathrm{~kg})$.

Aos 60 DAE foram avaliadas: altura de planta $(\mathrm{H})$ - determinada da superfície do substrato até o meristema apical, com auxílio de régua graduada $(\mathrm{cm})$, enquanto ao diâmetro do coleto (DC), mediu-se por meio de um paquímetro digital. Após as avaliações morfológicas, retirou-se as plantas dos vasos, cortando-se o caule rente ao solo, separando a parte aérea. As raízes foram separadas pelo processo de lavagem com água até separá-las completamente do solo.

Os materiais vegetais, parte aérea e raízes foram pesados em balança analítica de precisão $(0,01 \mathrm{~g})$ para estimar a biomassa da parte aérea (BMPA) e biomassa da raiz (BMR). Posteriormente, o material vegetal foi seco em estufa a $65^{\circ} \mathrm{C} \mathrm{com}$ circulação forçada de ar até atingir peso constante, sendo pesada em balança analítica de precisão para avaliação da massa seca 
da parte aérea (MSPA) e massa seca de raízes (MSR). A partir destas, calculou-se os índices morfológicos: o quociente de robustez (ROB) que foi determinado em função da relação entre altura da planta e o diâmetro do coleto (H/DC) e, o índice de qualidade de Dickson (IQD), por meio da Equação 1, proposta por (Dickson, Leaf e Hosner, 1960):

$$
\mathrm{IQD}=\frac{\mathrm{MST}}{\left[\left(\frac{\mathrm{H}}{\mathrm{DC}}\right)+\left(\frac{\mathrm{MSPA}}{\mathrm{MSR}}\right)\right]}
$$

Eq. 1

Onde: MST é a massa seca total (g); H é a altura da parte aérea $(\mathrm{cm})$; DC é o diâmetro do coleto (mm); MSPA é a massa seca da parte aérea (g); e, MSR é a massa seca das raízes (g).

Os dados experimentais foram submetidos à análise de variância (ANOVA), com a aplicação do teste $\mathrm{F}$ a $5 \%$ de probabilidade, e quando significativo, aplicou-se teste Tukey a 5\% de probabilidade para o agrupamento das médias, com auxílio do software Rbio (Bhering, 2017). Os resultados de biomassa e massa seca de raiz de leucena foram transformados por raiz de X. Os dados quantitativos quando significativos pela ANOVA foram analisados por regressão.

Os resultados para os parâmetros morfológicos foram avaliados por rede de correlações de Pearson $(P>0,6)$. A rede de correlação foi utilizada para expressar graficamente os resultados, nos quais a proximidade entre os nós (traços) é proporcional aos valores de correlação absoluta entre os parâmetros avaliados. A espessura da borda foi controlada pela estimativa das correlações, onde as correlações positivas foram destacadas em verde, enquanto as correlações negativas foram representadas em vermelho. Esta rede foi realizada através do software Rbio (Bhering, 2017).

\section{Resultados e Discussão}

A altura de planta e diâmetro do caule da leucena não foram influenciadas significativamente $(P>0,05)$ pelo uso de fontes e doses de fósforo utilizadas (Tabela 1). Conforme a Tabela 1, ao observarmos os valores de $\mathrm{F}$ calculado, as fontes e doses de fósforo promoveram alterações nos parâmetros de BMPA, BMR, MSPA e MSR da leucena, porém sem interações significativas entre fontes e doses. O quociente de robustez (ROB) da leucena foi influenciado significativamente somente para doses de fósforo. 
Tabela 1. Características de mudas de leucena em função de doses de fertilizante organomineral (OM) e mono-amônio-fosfato (MAP).

\begin{tabular}{|c|c|c|c|c|c|c|c|c|c|}
\hline Fontes & Dose de P & $\mathrm{AP}^{1}$ & $\mathrm{DC}$ & BMPA & BMR & MSPA & MSR & ROB & IQD \\
\hline & $\mathrm{mg} \mathrm{kg}^{-1}$ & $\mathrm{~cm}$ & $\mathrm{~mm}$ & ------ & $----g$ & $\mathrm{raso}^{-1}-{ }_{-}$ & ------- & & \\
\hline & 0 & 17,27 & 2,93 & 4,27 & 3,60 & 0,77 & 0,43 & 5,89 & 0,12 \\
\hline \multirow[t]{4}{*}{$\mathrm{OM}$} & 10 & 20,53 & 2,77 & 7,03 & 6,50 & 1,3 & 0,87 & 7,57 & 0,23 \\
\hline & 20 & 17,40 & 2,17 & 5,57 & 6,20 & 1,07 & 0,90 & 7,87 & 0,14 \\
\hline & 30 & 15,00 & 2,50 & 4,37 & 4,80 & 1,07 & 0,63 & 7,97 & 0,20 \\
\hline & 0 & 12,37 & 2,47 & 3,73 & 2,30 & 0,83 & 0,20 & 4,98 & 0,10 \\
\hline \multirow[t]{3}{*}{ MAP } & 10 & 19,53 & 2,50 & 7,40 & 3,23 & 1,20 & 0,43 & 7,30 & 0,20 \\
\hline & 20 & 21,90 & 3,07 & 8,17 & 3,60 & 1,63 & 0,50 & 6,13 & 0,22 \\
\hline & 30 & 22,00 & 3,43 & 8,23 & 2,67 & 1,70 & 0,43 & 6,90 & 0,17 \\
\hline${ }^{2}$ Média OM & & 17,55 & 2,59 & $5,31^{b}$ & $5,28^{a}$ & $1,05^{b}$ & $0,71^{a}$ & 7,32 & 0,18 \\
\hline Média MAP & & 18,95 & 2,87 & $6,88^{a}$ & $2,95^{\mathrm{b}}$ & $1,34^{\mathrm{a}}$ & $0,39^{b}$ & 6,33 & 0,17 \\
\hline C.V. $(\%)$ & & 21,28 & 21,37 & 24,77 & 15,51 & 15,51 & 15,08 & 24,47 & 33,46 \\
\hline Fontes de variação & & \multicolumn{8}{|c|}{${ }^{2}$ Valores de $F$ calculado } \\
\hline Fertilizantes & & $0,77^{\mathrm{ns}}$ & $1,33^{\mathrm{ns}}$ & $6,53^{*}$ & $4,63^{*}$ & $5,00 *$ & $3,00 *$ & $0,03^{\text {ns }}$ & $0,06^{\mathrm{ns}}$ \\
\hline Doses & & $2,25^{\mathrm{ns}}$ & $0,46^{\mathrm{ns}}$ & $5,51 *$ & $8,40 *$ & $4,28 *$ & $1,70^{*}$ & $2,48 *$ & $0,17^{\mathrm{ns}}$ \\
\hline Fertilizantes x Doses & & $2,86^{\mathrm{ns}}$ & $2,45^{\mathrm{ns}}$ & $2,67^{\mathrm{ns}}$ & $0,35^{\mathrm{ns}}$ & $1,94^{\mathrm{ns}}$ & $0,05^{\mathrm{ns}}$ & $0,34^{\mathrm{ns}}$ & $0,49^{\text {ns }}$ \\
\hline
\end{tabular}

${ }^{1} \mathrm{AP}$ : altura de planta; DC: diâmetro do caule; BMPA: biomassa da parte aérea; BMR: biomassa de raiz; MSPA: massa seca da parte aérea; MSR: massa seca de raiz; ROB: quociente de robustez; IQD: índice de qualidade de Dickson. ${ }^{2}$ Médias das fontes de fertilizantes seguidas de mesma letra minúscula nas colunas pertencem ao mesmo grupo conforme o teste de Tukey $(P \leq 0,05) .{ }^{3}$ ns: não significativo; * Significativo pelo teste F $(P<0,01)$; Fonte: Autores.

A altura de mudas e o diâmetro do caule são parâmetros utilizados por viveiros florestais no momento da seleção de mudas, permitindo selecionar apenas as plantas que apresenta tamanho ideal para espécie antes de ser levada para campo (Oliveira et al., 2016). Estudos realizados por Faria et al., (2016), verificaram resultados significativos em altura em mudas de Mimosa setosa B., quando utilizado 25\% de substrato comercial, 35\% de cama de aviário e 40\% de solo (Latossolo vermelhoamarelo). Porém, Dantas et al., (2016), não observou incremento na altura de plantas de leucena com o fornecimento de nutrientes através de solução nutritiva, devido à baixa exigência nutricional no início de seu desenvolvimento. Desta forma, a altura de plantas e o diâmetro do caule pode não responder a adubação em mudas devido ao estádio inicial das plantas.

A BMPA apresentou regressão polinomial quadrática significativa para doses de MAP (Figura 1A). Aplicando-se a dose 22,77 $\mathrm{mg} \mathrm{kg}^{-1}$ de $\mathrm{P}_{2} \mathrm{O}_{5}$, obteve-se o máximo incremente de 7,94 $\mathrm{g} \mathrm{vaso}^{-1}$ de BMPA. Andreazza et al., (2013) observaram que a adição de $25 \%$ de vermicomposto no substrato incrementa a BMPA de leucena, o vermicomposto utilizado possuía 8,2 g $\mathrm{kg}^{-1}$ de $\mathrm{N}, 4 \mathrm{~g} \mathrm{~kg}^{-1}$ de P e 8,0 $\mathrm{g} \mathrm{kg}^{-1}$ de K. Porém, a fonte mineral (MAP) proporcionou maior média de BMPA (6,88 g vaso-1) (Tabela 1), devido ser mais solúvel que a fonte organomineral e promover a rápida liberação de $\mathrm{N}$ e $\mathrm{P}$ para a planta, favorecendo o crescimento da parte aérea da leucena.

As doses de OM promoveram incrementos produtivos de BMR apresentando uma curva de resposta com equação polinomial quadrática significativa (Figura 1B). Pela derivada de equação, verificou-se que a dose $16 \mathrm{mg} \mathrm{kg}^{-1}$ de $\mathrm{P}_{2} \mathrm{O}_{5}$, promoveu o incremento máximo de $5 \mathrm{~g} \mathrm{vaso}^{-1}$ de BMR em mudas de leucena. A maior média de $\mathrm{BMR}\left(5,28 \mathrm{~g} \mathrm{vaso}{ }^{-1}\right)$, foi proporcionado pela adição do fertilizante $\mathrm{OM}\left(6,28 \mathrm{~g} \mathrm{vaso}^{-1}\right)$. O uso de fontes orgânicas que tem $\mathrm{P}$ menos solúvel promove o crescimento radicular, pois a planta necessita emitir maior quantidade de raízes para absorver o P que imóvel no substrato. A maior concentração de raízes no substrato pode favorecer o pegamento de mudas no campo (Dantas et al., 2016). 
Figura 1. Biomassa da parte aérea (BMPA) (A) e de biomassa de raiz (BMR) (B) de mudas de leucena em função das doses fósforo.
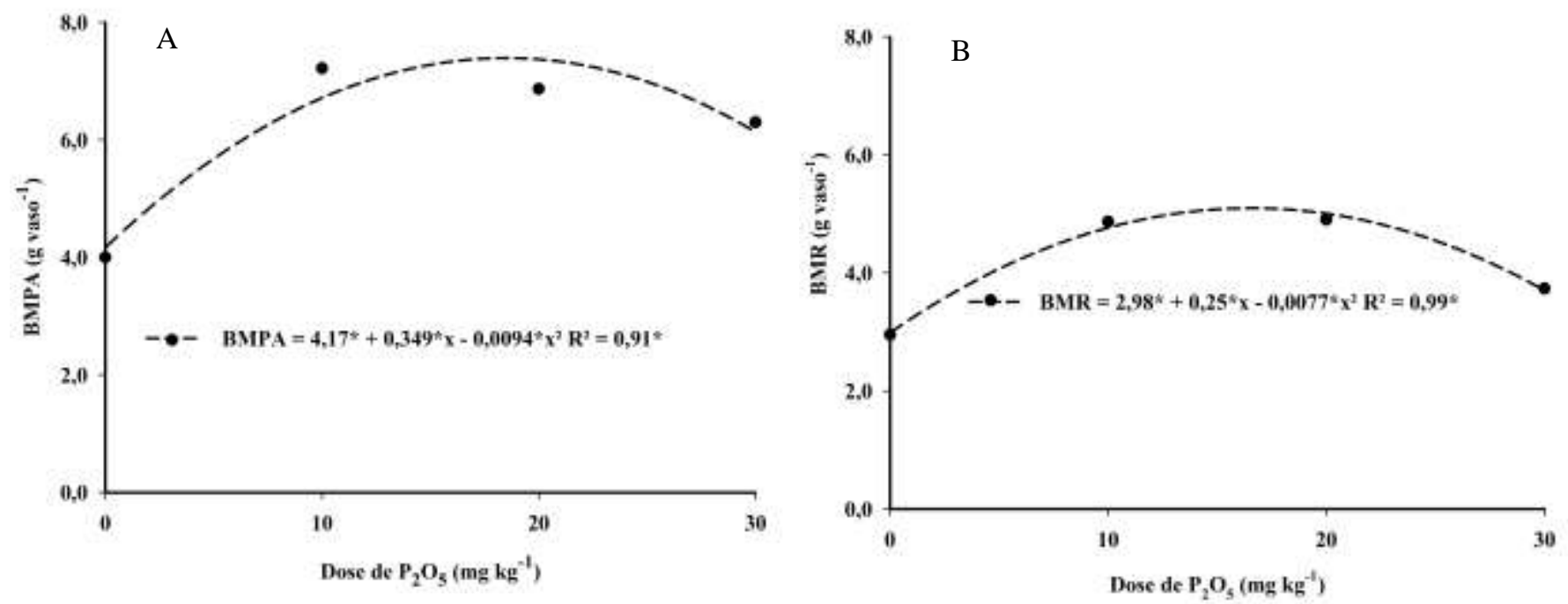

*Equação significativa pelo teste $\mathrm{F}(P<0,01)$.

Fonte: Autores

O incremento de MSPA é proporcional a doses crescentes de fósforo, apresentando crescimento linear (Figura 2A). A média de MSPA apresentou incremento de $21 \%$ para o uso de MAP quando comparado ao ORG (Tabela 1). Dantas et al. (2016), avaliaram a fertirrigação por capilaridade com o uso de diferentes soluções nutritivas e, verificaram que para o incremento de MSPA deve-se adicionar P no substrato das mudas de leucena. Para MSR, verificou-se que doses de fósforo promoveram incrementos de MSR apresentando uma curva de resposta com equação polinomial quadrática significativa

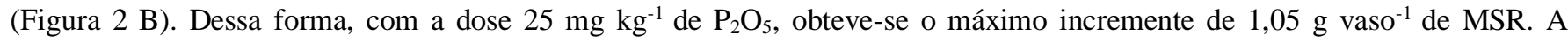
adubação fosfatada utilizando como fonte OM promoveu o incremento de 54\% na média de MSR em mudas de leucena quando comparado a adubação com MAP (Tabela 1).

Plantas com sistema radicular menos desenvolvido apresenta maior capacidade de absorção de fósforo quando comparado com raízes de plantas mais velhas que apresenta menor capacidade de absorção (Prado et al., 2004). Incremento em massa seca da raiz de mudas de teca (Tectona grandis Linn. F.) foi encontrado por Trazzi et al. (2013), obtendo-se 0,69 a 2,07 g planta ${ }^{-1}$, apresentando a maior média, respectivamente para o tratamento que se utilizava $25 \%$ de substrato comercial, $40 \%$ de solo e 35\% de cama de frango. Araújo et al. (2017) também encontraram incremento MSR avaliando mudas de Paricá (Schizolobium amazonicum) com o uso de substratos orgânicos. O ganho em matéria seca com a fonte organomineral é explicado pela mineralização da cama de frango, permitindo o fornecimento de $\mathrm{NH}_{3}, \mathrm{NO}^{3-}, \mathrm{H}_{2} \mathrm{PO}^{4-}, \mathrm{HPO}_{4}{ }^{2-}$ e $\mathrm{SO}_{4}{ }^{2-}$, sendo assimilado pelas plantas (Moreira e Siqueira, 2006). 
Figura 2. Massa seca da parte aérea (MSPA) (A) e massa seca de raiz (MSR) (B) em função das doses de fósforo.
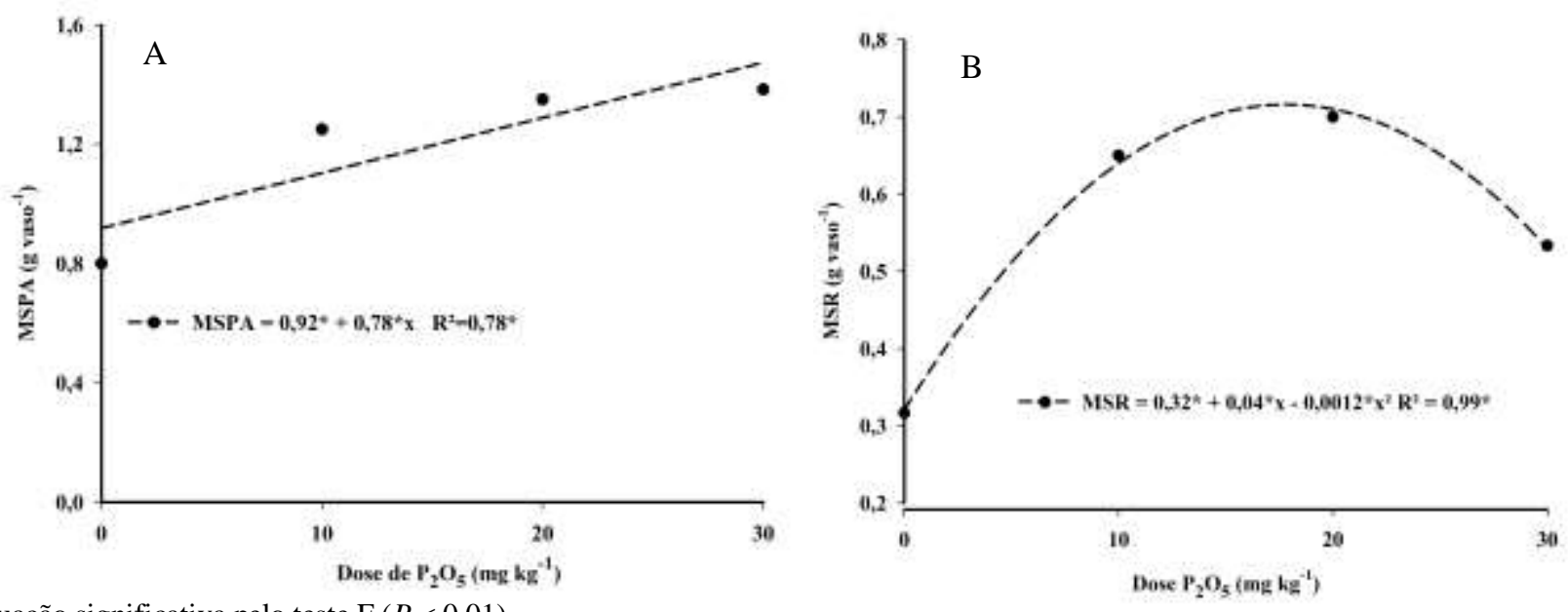

*Equação significativa pelo teste $\mathrm{F}(P<0,01)$.

Fonte: Autores

O quociente de robustez (ROB) apresentou efeito exponencial para as doses de fósforo (Figura 3). Dessa forma, pela derivada da equação exponencial, foi possível verificar que partir da dose $2,4 \mathrm{mg} \mathrm{kg}^{-1}$, obteve-se a máxima robustez da muda de leucena. A robustez indica que a muda está mais adaptável ao plantio no campo (Zuffo et al., 2018). Além disso, as médias de ROB de mudas de leucena (Tabela 1) apresentaram valores abaixo de 10, ideal para mudas de boa qualidade, pois ROB acima de 10 é característico de plantas estioladas (Melo et al., 2018). Nesse sentido, deve-se fornecer a doses acima de 2,4 mg $\mathrm{kg}^{-1}$ de $\mathrm{P}_{2} \mathrm{O}_{5}$ para melhorar a qualidade da muda de leucena, e favorecer seu desenvolvimento a campo.

Figura 3. Quociente de Robustez de mudas de leucena em função de doses de fósforo.

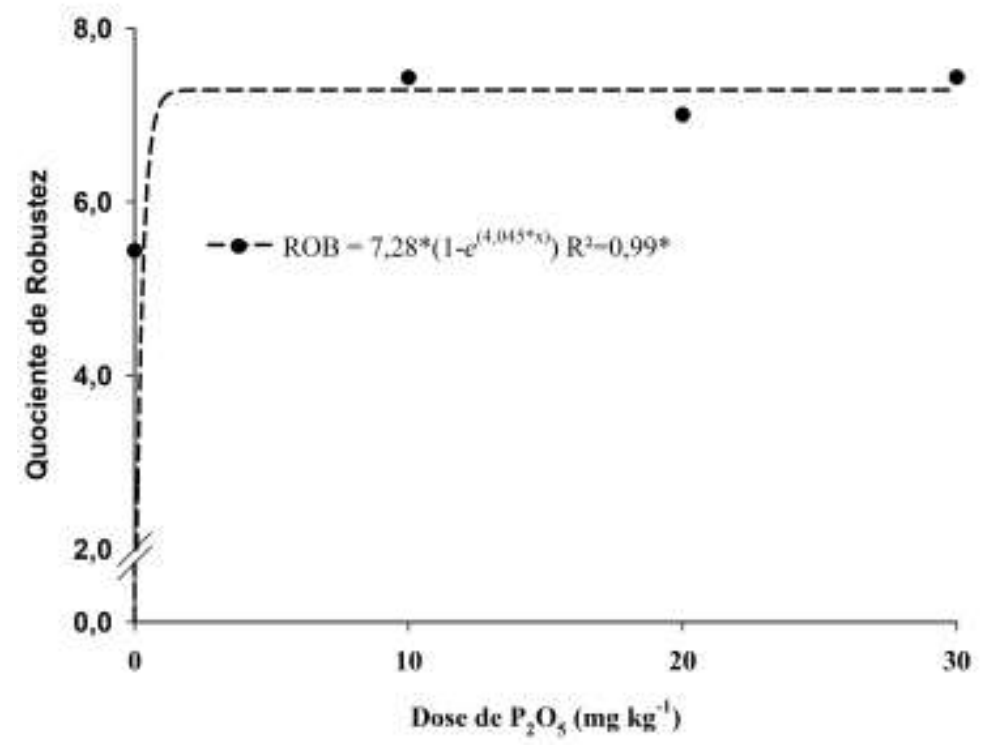

*Equação significativa pelo teste $\mathrm{F}(P<0,01)$.

Fonte: Autores

Os parâmetros AP, DC, BMPA, BMR, MSPA, MSR, ROB e IQD das mudas de leucena correlacionam positivamente entre si pela correlação de Pearson's $(P>0,60)$ quando submetidas a adubação fosfatada (Figura 4). Dessa forma, fertilizante 
que favorecem o desenvolvimento radicular da planta, neste caso o OM, melhoram o IQD da muda, e os fertilizantes que favorecem o desenvolvimento da parte aérea da planta, como o MAP, proporcionam melhor robustez. Entretanto, observou-se que correlações negativas entre DC e ROB; DC e MSR; e ROB e MSPA, de mudas de leucena submetida a adubação fosfatada. Deve-se observar que o coeficiente de robutez (ROB) é a divisão do valor de altura de planta (AP) e pelo valor do diâmetro do caule (DC), assim o aumento do DC diminui consequentemente a ROB (Gomes et al., 2002).

Figure 4. Rede de correlação dos componentes morfológicos de Leucena submetida a doses de fertilizante organomineral e mineral. (Pearson's $P>0.60$ ).

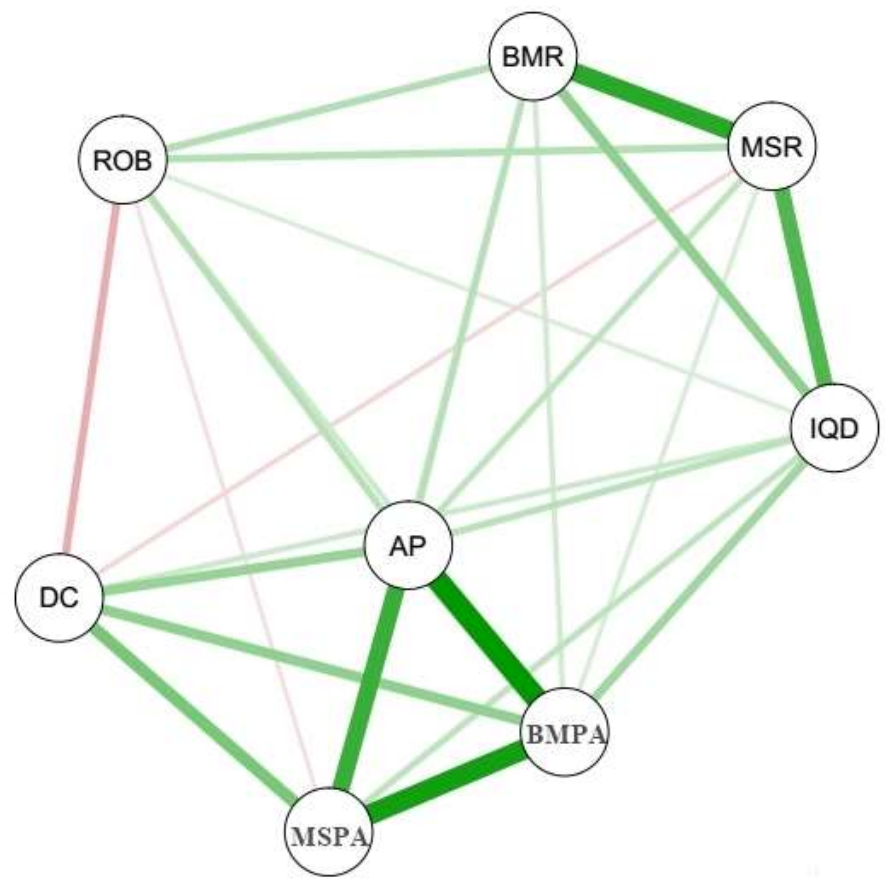

AP: altura de planta; DC: diâmetro do caule; BMPA: biomassa da parte aérea; BMR: biomassa de raiz; MSPA: massa seca da parte aérea; MSR: massa seca de raiz; ROB: índice de robustez; IQD: índice de qualidade de Dickson. Fonte: Autores

As médias de IQD das mudas de leucena foram de 0,18 para o uso de fertilizante organomineral (OM) e 0,17 para o uso de fertilizante mineral (MAP) (Tabela 1). Para, Gomes e Paiva (2004), mudas de boa qualidade apresenta valor de IQD acima de 0,2. Nesse sentido observou-se que doses de 10 e $30 \mathrm{mg} \mathrm{kg}^{-1}$ de $\mathrm{P}_{2} \mathrm{O}_{5}$ utilizando como fonte $\mathrm{OM}$, e 10 e $20 \mathrm{mg} \mathrm{kg}^{-1} \mathrm{de}$ $\mathrm{P}_{2} \mathrm{O}_{5}$ utilizando como fonte MAP, proporciona médias de IQD acima de 0,20 para mudas de leucena. Dessa forma, uma dose adequada de fertilizantes fosfatados pode melhorar a qualidade de mudas de leucena. Para Oliveria et al. (2016) e Dantas et al. (2016) existe a necessidade de associar IQD com outro parâmetro de avaliação de qualidade de mudas. Assim, efetivamente verificou-se IQD de mudas de leucena submetidas a adubação fosfatada tem correlação positiva com os parâmetros AP, DC, BMPA, BMR, MSPA, MSR e ROB.

As mudas de leucena submetidas a dose de acima de $2,4 \mathrm{mg} \mathrm{kg}^{-1} \mathrm{P}_{2} \mathrm{O}_{5}$ apresentam maior ROB, e que $25 \mathrm{mg} \mathrm{kg}^{-1} \mathrm{P}_{2} \mathrm{O}_{5}$, favorece a produção MSR, independente da fonte $\mathrm{P}$ utilizada. Contudo, observou-se também que o MAP promove maior média de MSPA e o ORG promove maior MSR. Neste contexto, verifica-se a necessidade de fornecimento de doses adequadas dos dois fertilizantes fosfatados para obter-se uma muda de leucena de alta qualidade.

\section{Conclusão}

A qualidade de mudas de leucena é influenciada pelo uso de diferentes fontes e doses de fósforo. 
As maiores médias de massa seca da parte aérea de mudas leucena são proporcionadas pelo uso de fertilizante mineral, e as maiores médias de massa seca de raiz são proporcionadas pelo uso de fertilizante organomineral, utilizando como fonte orgânica, cama de aviário.

O quociente de robustez e índice de qualidade de Dickson de mudas de leucena estão correlacionados positivamente com AP, DC, BMPA, BMR, MSPA, MSR.

\section{Agradecimentos}

O presente trabalho foi realizado com apoio da Coordenação de Aperfeiçoamento de Pessoal de Nível Superior Brasil (CAPES) - Código Financiamento 001, da Universidade Federal do Piauí e da Universidade Federal de Mato Grosso do Sul.

\section{Referências}

Almeida Júnior, A. B. de, Nascimento, C. W. A. do, Sobral, M. F., Silva, F. B. V. da, \& Gomes, W. A. (2011). Soil fertility and uptake of nutrients by sugarcane fertilized with filter cake. Revista Brasileira de Engenharia Agricola e Ambiental, 15(10), 1004-1013. https://doi.org/10.1590/S141543662011001000003

Alvares, C. A., Stape, J. L., Sentelhas, P. C., Gonçalves, J. L. M., \& Sparovek, G. (2014). Köppen's climate classification map for Brazil. Meteorologische Zeitschrift, 22(6), 711-728. https://doi.org/10.1127/0941-2948/2013/0507

Andreazza, R., Antoniolli, Z. I., Ferreira, R., Schirmer, G. K., Scheid, D. L., Quadro, M. S., \& Barcelos, A. A. (2013). Efeito de vermicomposto no crescimento de ipê amarelo (Handroanthus chrysotrichus) e leucena (Leucaena leucocephala) em solo arenoso. Nativa, 1(1), 29-33. https://doi.org/10.14583/2318-7670.v01n01a06

Araújo, E. F., Aguiar, A. S., Arauco, A. M. de S., Gonçalves, E. de O., \& Almeida, K. N. S. de. (2017). Crescimento e qualidade de mudas de paricá produzidas em substratos à base de resíduos orgânicos. Nativa, 5(1), 16-23. https://doi.org/10.5935/2318-7670.v05n01a03

Baron, R., Benitez, I. C., \& Gonzalez, J. L. (1995). Influência de la dosis creciente deun abono orgánico en un cultivo de trigo. Agrochimica, $39,280-289$.

Bhering, L. L. (2017). Rbio: A tool for biometric and statistical analysis using the R platform. Crop Breeding and Applied Biotechnology, 17(17), 187-190. https://doi.org/10.1590/1984

Corrêa, R. M., Nascimento, C. W. A. D., Souza, S. K. D. S., Freire, F. J., \& Silva, G. B. D. (2005). Gafsa rock phosphate and triple superphosphate for dry matter production and P uptake by corn. Scientia Agricola, 62(2), 159-164.

Dantas, R. D. P., Pereira, K. T. O., Cavalcante, A. L. G., Souza, A. A. T., Souza Neta, M. L. de, \& Oliveira, F. D. A. de. (2016). Fertirrigação por capilaridade em mudas de Leucaena leucocephala (Lam.) de Wit. Revista Agro@ Mbiente On-Line, 10(2), 161. https://doi.org/10.18227/1982-8470ragro.v10i2.3202

Decker, V., Klosowski, É. S., Malavasi, U. C., \& Nunes, A. (2011). Evaluation of the Effects of Light Intensities Upon the Initial Development. Ciência Floresta, 21(4), 609-618.

Dickson, A., Leaf, A. L., \& Hosner, J. F. (1960). Quality appraisal of white spruce and white pine seedling stock in nurseries. The Forestry Chronicle, 36(1), $10-13$.

Faria, J. C. T., Caldeira, M. V. W., Delarmelina, W. M., \& Rocha, R. L. F. (2016). Substratos alternativos na produção de mudas de Mimosa setosa Benth. Ciencia Florestal, 26(4), 1075-1086. https://doi.org/10.5902/1980509824996

Gomes, J. M., Couto, L., Leite, H. G., Xavier, A., \& Garcia, S. L. R. (2002). Parâmetros morfológicos na avaliação da qualidade de mudas de Eucalyptus grandis. Revista Árvore, 26(6), 655-664. https://doi.org/10.1590/S0100-67622002000600002

Gomes, J. M., \& Paiva, H. N. (2004). Viveiros florestais: propagação sexuada. Viçosa, MG.

Medeiros, J. D. (1992). Reflorestar é preservar. Florianópolis: Souza Cruz.

Melo, L. A. de, Abreu, A. H. M. de, Leles, P. S. dos S., Oliveira, R. R., \& Silva, D. T. (2018). Qualidade e crescimento inicial de mudas de Mimosa caesalpiniifolia Benth produzidas em diferentes volumes de recipientes. Ciência Florestal, 28(1), 47-55.

Moreira, F. M. S., \& Siqueira, J. O. (2006). Microbiologia e bioquímica do solo. Lavras.

Oliveira, M. C., Ogata, R. S., Andrade, G. A. de, Santos, D. da S., Souza, R. M., Guimarães, T. G., Ribeiro, J. F. (2016). Manual de Viveiro e produção de mudas: espécies arbóreas nativas do cerrado. Brasília: Rede de Sementes do cerrado.

Prado, R. M., Braghirolli, L. F., Natale, W., Corrêa, M. C. M., \& Almeida, E. V. (2004). Potassium application on the nutritional status and dry matter production of passion fruit cutting. Revista Brasileira de Fruticultura, 26(2), 295-299.

RAij, B. V. (2011). Fertilidade do solo e manejo de nutrientes. (Institute, I. P. N., Ed.). Piracicaba. 
Research, Society and Development, v. 10, n. 2, e28710212095, 2021

(CC BY 4.0) | ISSN 2525-3409 | DOI: hittp://dx.doi.org/10.33448/rsd-v10i2.12095

Santos, L. C. da S., Costa, J. P., Vale, V. S., \& Dias Neto, O. C. (2019). Resposta de espécies arbóreas nativas de Cerrado à fertilização com diferentes substratos. Advances in Forestry Science, 6(1), 487-493. https://doi.org/10.34062/afs.v6i1.5532

Silva, F. S. (2011). Manual de análises de solos, plantas e fertilizantes. Embrapa.

Silva, M. D. A., Souza, C. M. S., Pinto, A. P., Pompeu, R. C. F. F., Silva, D. S., Coutinho, M. J. F., \& Fontenele, R. M. (2015). Avaliação da composição químico-bromatológica das

silagens de forrageiras lenhosas do semiárido brasileiro. Semina: Ciências Agrárias, 36(1), 571-577.

Sousa, B. F. (2005). Circular técnica 18: Leucena: Produção e Manejo no Nordeste Brasileiro. (Caprinos, E., Ed.). Sobral, CE.

Sousa, D. M. G., \& Lobato, E. (2004). Cerrado: correção do solo e adubação (2a). Brasília: Embrapa Informação Tecnológica.

Taiz, L., Zeiger, E., Moller, I. M., \& Murphy, A. (2017). Fisiologia e desenvolvimento vegetal. Artmed.

Tejada, M., Benitez, C., \& Gonzalez, J. L. (2002). Nitrogen mineralization in soil with conventional and organomineral fertilization practices. Communications in Soil Science and Plant Analysis, 33(19-20), 3679-3702. https://doi.org/10.1081/CSS-120015915

Trazzi, P. A., Caldeira, M. V. W., Passos, R. R., \& Gonçalves, E. de O. (2013). Substratos de origem orgânica para produção de mudas de teca (Tectona grandis Linn. F.). Ciencia Florestal, 23(3), 401-409.

Zuffo, A. M., Setiener, F., Zuffo Júnior, J. M., \& Santos, D. M. da S. (2018). Tamanho de recipientes na formação de mudas de Peltophorum dubium (Sprengel) Taubert (Fabaceae). Revista Engenharia Na Agricultura, 26(4), 322-333. https://periodicos.ufv.br/ojs/reveng/article/view/134/111 